\title{
Remodeling the Vascular Microenvironment of Glioblastoma with $\alpha$-Particles
}

\author{
Katja Behling ${ }^{1}$, William F. Maguire ${ }^{2}$, Valentina Di Gialleonardo ${ }^{1}$, Lukas E.M. Heeb ${ }^{1}$, Iman F. Hassan ${ }^{1}$, Darren R. Veach ${ }^{1}$, \\ Kayvan R. Keshari ${ }^{1}$, Philip H. Gutin ${ }^{3,4}$, David A. Scheinberg ${ }^{2,5}$, and Michael R. McDevitt ${ }^{1,6}$ \\ ${ }^{I}$ Department of Radiology, Memorial Sloan Kettering Cancer Center, New York, New York; ${ }^{2}$ Department of Molecular Pharmacology, \\ Memorial Sloan Kettering Cancer Center, New York, New York; ${ }^{3}$ Department of Neurosurgery, Memorial Sloan Kettering Cancer \\ Center, New York, New York; ${ }^{4}$ Department of Neurological Surgery, Weill Cornell Medical College, New York, New York; ${ }^{5}$ Department \\ of Pharmacology, Weill Cornell Medical College, New York, New York; and ${ }^{6}$ Department of Medicine, Weill Cornell Medical College, \\ New York, New York
}

\begin{abstract}
Tumors escape antiangiogenic therapy by activation of proangiogenic signaling pathways. Bevacizumab is approved for the treatment of recurrent glioblastoma, but patients inevitably develop resistance to this angiogenic inhibitor. We previously investigated targeted a-particle therapy with ${ }^{225} \mathrm{Ac}-\mathrm{E} 4 \mathrm{G} 10$ as an antivascular approach and showed increased survival and tumor control in a highgrade transgenic orthotopic glioblastoma model. Here, we investigated changes in tumor vascular morphology and functionality caused by ${ }^{225}$ Ac-E4G10. Methods: We investigated remodeling of the tumor microenvironment in transgenic Ntva glioblastoma mice using a therapeutic 7.4-kBq dose of ${ }^{225} \mathrm{Ac}-\mathrm{E} 4 \mathrm{G} 10$. Immunofluorescence and immunohistochemical analyses imaged morphologic changes in the tumor blood-brain barrier microenvironment. Multicolor flow cytometry quantified the endothelial progenitor cell population in the bone marrow. Diffusion-weighted MR imaged functional changes in the tumor vascular network. Results: The mechanism of drug action is a combination of remodeling of the glioblastoma vascular microenvironment, relief of edema, and depletion of regulatory $\mathrm{T}$ and endothelial progenitor cells. The primary remodeling event is the reduction of both endothelial and perivascular cell populations. Tumor-associated edema and necrosis were lessened, resulting in increased perfusion and reduced diffusion. Pharmacologic uptake of dasatinib into tumor was enhanced after a-particle therapy. Conclusion: Targeted antivascular a-particle radiation remodels the glioblastoma vascular microenvironment via a multimodal mechanism of action and provides insight into the vascular architecture of platelet-derived growth factor-driven glioblastoma.
\end{abstract}

Key Words: glioblastoma; vascular endothelium; pericytes; radioimmunotherapy; actinium-225; ${ }^{225} \mathrm{Ac}$

J Nucl Med 2016; 57:1771-1777

DOI: $10.2967 /$ jnumed.116.173559

\section{A} cer therapy. Bevacizumab is now the standard of care for treatment of colon, lung, kidney, and brain cancer (1). Tumors require a blood

\footnotetext{
Received Feb. 1, 2016; revision accepted Apr. 26, 2016.

For correspondence or reprints contact: Michael R. McDevitt, Department of Radiology, Memorial Sloan Kettering Cancer Center, 1275 York Ave., Box 231, New York, NY 10065

E-mail: m-mcdevitt@ski.mskcc.org

Published online Jun. 3, 2016.

COPYRIGHT (C 2016 by the Society of Nuclear Medicine and Molecular Imaging, Inc.
}

supply to grow larger than 1-2 $\mathrm{mm}^{3}$ (2); therefore, neovascularization and angiogenesis have critical roles in growth and metastasis, making tumor vasculature a strategic target. Therapies target fundamental architectural and functional differences between tumor tissue and healthy vascular tissue (3). Normal vascular networks exhibit a hierarchical organization of evenly distributed vessels allowing adequate perfusion. In contrast, an aggressive tumor overexpresses proangiogenic factors (e.g., vascular endothelial growth factor [VEGF]), resulting in chaotic and aberrant vessel growth and a disorganized vascular labyrinth. Geometric resistance caused by this microenvironment leads to impaired blood flow and reduced functionality.

Therapeutic mechanisms that interfere with the VEGF/VEGF receptor signaling axis by blocking either VEGF-A (bevacizumab) or VEGF receptors 1 and 2 (sorafenib and sunitinib (4)) have to be chronically administered, and tumors often develop resistance by upregulating other signaling pathways (5). Alternative antivascular approaches employ vascular disrupting agents (6) or use radioimmunotherapy to target epitopes exclusively expressed by tumor neovasculature (7-10). Targeted $\alpha$-particle therapy deposits a high dose of energy (5-8 MeV) over a short range $(50-80 \mu \mathrm{m})$, producing specific and potent cytotoxicity. $\alpha$-particle-emitting radionuclides are exceptionally potent therapeutic effectors; a single traversal of a high-linear energy transfer $\alpha$-particle through a cell can be lethal $(7,8)$. Moreover, $\alpha$-particle ranges match vascular diameters $(10 \mu \mathrm{m}<$ vessel $<100 \mu \mathrm{m})$.

We developed ${ }^{225} \mathrm{Ac}-\mathrm{E} 4 \mathrm{G} 10$ as an antivascular radioimmunotherapy that specifically targets tumor vasculature $(9,10) .{ }^{225} \mathrm{Ac}$ is an $\alpha$-emitting radionuclide that, together with its several daughters, can deposit up to 4 net $\alpha$-emissions at the targeted site. E4G10 is a monoclonal antibody directed against monomeric vascular endothelial cadherin expressed on tumor neovasculature (11) and endothelial progenitor cells (EPCs) (12). Monomeric vascular endothelial cadherin is dimerized in resting vasculature; thus, the targeted epitope is concealed from E4G10.

${ }^{225}$ Ac-E4G10 has been reported to have potent efficacy against carcinomas $(9,10)$ and glioblastoma (13). Glioblastomas are histologically distinct from lower-grade tumors (14). Glioblastoma vessels are poorly perfused and highly permeable, leading to hypoxic tumor zones and brain parenchyma edema (15), as well as breakdown of the blood-brain barrier (BBB) (16). Despite encouraging effects from bevacizumab, patients inevitably relapse with more aggressive, infiltrative, and treatment-resistant disease (17). 
Our preclinical studies on the use of ${ }^{225} \mathrm{Ac}-\mathrm{E} 4 \mathrm{G} 10$ as an alternative antivascular approach in transgenic glioblastoma mice showed therapeutic activity as monotherapy and a significantly increased survival (13). Here, we investigated the microenvironment architecture and function in remodeled tumor resulting from $\alpha$-particle irradiation. We present a multimodal-mechanism-of-action study in which ${ }^{225}$ Ac-E4G10 remodels the glioblastoma vascular microenvironment in a disease-relevant transgenic glioblastoma mouse model.

\section{MATERIALS AND METHODS}

\section{Radiochemistry}

E4G10 (rat anti-mouse, anti-monomeric vascular endothelial cadherin antibody; Eli Lilly and Co.) and isotype-matched control (IgG2a, rat antimouse anti-keyhole limpet hemocyanin; R\&D Systems) were labeled as previously described $(18,19)$ with ${ }^{225} \mathrm{Ac}$ (ORNL).

\section{Mouse Model and Induction of Glioblastoma}

Nestin-tumor virus A (Ntva) mice (Ink4a-Arf ${ }^{-1-}$; FVB/N, C57BL6, and BALB/C background) with the retroviral RCAS (replication-competent avian sarcoma-leukosis virus) system (20) were used. The RCAS vector carries the tumor-driver human platelet-derived growth factor (PDGF) B. Tumor was induced by stereotactic intracranial surgery as previously described (13).

\section{Mechanistic Study Design}

Glioblastoma-bearing Ntva mice were imaged with T2-weighted MRI at $4.5 \mathrm{wk}$ after induction to assess tumor size and were placed into treatment groups according to tumor size (13). Anesthetized mice (1.5\% isoflurane) received a single $7.4-\mathrm{kBq}(200 \mathrm{nCi})(0.10 \mathrm{~mL})$ dose of ${ }^{225} \mathrm{Ac}-\mathrm{E} 4 \mathrm{G} 10,{ }^{225} \mathrm{Ac}$-isotype control, or $1 \%$ human serum albumin (vehicle) via the retroorbital venous plexus on day 0 . Ten days after treatment, the animals were either placed in the dasatinib study or evaluated by diffusion-weighted MRI to assess functional transport parameters and subsequently euthanized to harvest tumors for histologic analysis and bone marrow for EPC-population analysis.

\section{Immunohistochemistry and Immunofluorescence Imaging}

The brains and tumors of treated Ntva mice ( 4 per group) were fixed and sectioned for immunohistochemistry and immunofluorescence analysis $10 \mathrm{~d}$ after the antivascular treatment. Briefly, the mice were sacrificed, and tumor (including brain) was excised and fixed in $4 \%$ paraformaldehyde/phosphate-buffered saline for $48 \mathrm{~h}$. Fixed tissue was paraffin-embedded and cut into 5- $\mu \mathrm{m}$ sections. Immunohistochemistry and immunofluorescence staining were performed at the Memorial Sloan Kettering Cancer Center (MSKCC) Molecular Cytology Core Facility using a Discovery XT processor (Ventana Medical Systems).

Sections for immunohistochemistry were stained with anti-FoxP3 antibody $(0.5 \mu \mathrm{g} / \mathrm{mL}$; eBioscience), anti-Ki 67 antibody $(0.4 \mu \mathrm{g} / \mathrm{mL}$; Vector), or anti-HIF1a antibody ( $1 \mu \mathrm{g} / \mathrm{mL}$; Novus Bio). Sections were scanned using a Mirax digital slide scanner (Carl Zeiss Microimaging) with a $\times 20$ lens. Analysis was performed on whole-tumor sections with Pannoramic Viewer software (3DHISTECH).

Immunofluorescence staining was performed with unlabeled primary and labeled secondary antibodies. Sections were stained with antiCD31 for endothelium ( $1 \mu \mathrm{g} / \mathrm{mL}$; Dianova), anti-desmin for pericytes (2 $\mu \mathrm{g} / \mathrm{mL} ;$ Abcam), and anti-collagen IV for basement membrane $(1 \mu \mathrm{g} / \mathrm{mL}$; Serotec). Sections were counterstained with 4',6-diamidino2-phenylindole (DAPI, $5 \mu \mathrm{g} / \mathrm{mL}$; Sigma Aldrich). Stained slides were scanned with the Mirax digital slide scanner using a $\times 20$ lens and analyzed with Pannoramic Viewer software and MetaMorph software (Molecular Devices) for quantifications of whole-tumor sections. Confocal images of selected areas within the tumor were acquired with a TCS SP5 microscope (Leica Microsystems CMS GmbH) as $x, y, z$-scans under optimal sampling conditions. The data were deconvolved using AutoQuant X3 software (Media Cybernetics Inc.). Acquired data were segmented in 3 dimensions using Imaris software (Bitplane AG). To quantify ratios of single-vessel volume to associated pericyte volume, the data were first segmented and quantified for every single-vessel volume unit using Imaris software.

\section{Diffusion-Weighted MRI Study}

All diffusion-weighted MRI measurements of intravoxel incoherent motion (21) were performed on a permanent 1-T MRI system (nanoScan PET/MRI; Mediso). Tumor regions were identified on axial anatomic images acquired using a T2-weighted fast spin echo sequence (echo time/repetition time, $88.5 / 2,000 \mathrm{~ms}$ ). Five 3-mm slices were acquired with a $50-\mathrm{mm}$ field of view and a $256 \times 252$ matrix to cover the brain. Perfusion and diffusion data were acquired using a 2-dimensional echoplanar diffusion-weighted MRI sequence (echo time/repetition time, $48.7 / 1,000 \mathrm{~ms}$ ). Ten different b-values were applied, covering a range from 0 to $600 \mathrm{~s} / \mathrm{mm}^{2}(\mathrm{~b}=0,10,25,50,75,150,250,400$, 500 , and $600 \mathrm{~s} / \mathrm{mm}^{2}$ ); b-values less than $200 \mathrm{~s} / \mathrm{mm}^{2}$ cover predominantly perfusion events, and b-values greater than $200 \mathrm{~s} / \mathrm{mm}^{2}$ cover predominantly diffusion events. Slice thickness and field of view were chosen to match anatomic images. Variation in measured signal intensities due to breathing was decreased by measuring all b-values twice.

Osirix (Pixmeo) was used for data analysis. Axial T2-weighted fast spin echo images were used to localize the tumor. A region of interest (ROI) was manually drawn just within the tumor border and transferred to the echoplanar images. ROIs were also drawn on a healthy area of brain in the same section. The mean signal intensity from ROIs was reported and the data processed with Excel (Microsoft). Prism (GraphPad Software Inc.) was used to plot signal intensity (S), normalized by the mean signal intensity of the $b=0$ echoplanar image $\left(\mathrm{S}_{0}\right)$, against b-values. A biexponential model fitted the data to extract the perfusion coefficient $\left(D^{*}\right)$ and diffusion coefficient $(D)$ according to the following equation:

$$
\mathrm{S} / \mathrm{S}_{0}=(1-\mathrm{f}) \times \mathrm{e}^{-\mathrm{b} \times D}+\mathrm{f} \times \mathrm{e}^{-\mathrm{b} \times D *}
$$

The apparent $D^{*}$ (ADC) was extracted using a monoexponential fit:

$$
\mathrm{S} / \mathrm{S}_{0}=\mathrm{e}^{-\mathrm{b} \times \mathrm{ADC}}
$$

Curves were displayed as $\ln \left(\mathrm{S} / \mathrm{S}_{0}\right)$ versus b-value to determine their slope.

\section{Flow Cytometry}

Multicolor flow cytometry identified VEGF receptor 2-positive/ c-kit-positive/TER 119-negative/CD11b-negative cells in the marrow (22). Femur and tibia were dissected, and the red bone marrow was flushed with $2 \%$ fetal calf serum/Dulbecco modified Eagle medium and tumor removed for histopathologic examination. The red blood cells were depleted (Qiagen), and unspecific binding of antibodies to cells was blocked by incubating the cells with anti-mouse Fc-block (anti-CD16/32; BD Biosciences), 1:30 in FACS buffer (phosphate-buffered saline, $\mathrm{pH} 7.2 ; 0.5 \%$ bovine serum albumin; $2 \mathrm{mM}$ ethylenediaminetetraacetic acid), for $15 \mathrm{~min}$ at $4{ }^{\circ} \mathrm{C}$. The cells were subsequently stained by incubation at $4^{\circ} \mathrm{C}$ for $30 \mathrm{~min}$ with an antibody cocktail containing monoclonal rat-anti-mouse antibodies: Pacific blue-anti-CD11b $(0.5 \mu \mathrm{g} / \mathrm{mL}$; BD Biosciences), Pacific blueanti-TER 119 (5 $\mu \mathrm{g} / \mathrm{mL}$; Biolegend), Alexa Fluor 488-anti-c kit (4 $\mu \mathrm{g} / \mathrm{mL}$; BD Biosciences), and phycoerythrin-anti-VEGF receptor $2(2.5 \mu \mathrm{g} / \mathrm{mL}$; $\mathrm{BD}$ Biosciences). The cells were washed twice and measured in $0.2 \mathrm{~mL}$ of FACS buffer containing DAPI on an LSRII flow cytometer (BD Biosciences). Data processing and analysis used FlowJo 
software (Tree Star). An additional experiment compared the bone marrowEPC titer of untreated glioblastoma-bearing Ntva mice $(n=4)$ and naïve Ntva mice $(n=3)$.

\section{${ }^{14} \mathrm{C}$-Autoradiography of Dasatinib Uptake}

Ntva glioblastoma mice were treated with $7.4 \mathrm{kBq}$ of ${ }^{225} \mathrm{Ac}-\mathrm{E} 4 \mathrm{G} 10$ $(n=6)$ or vehicle $(n=5)$ and $10 \mathrm{~d}$ later received $111 \mathrm{kBq}(0.32 \mathrm{mg}$, $3 \mu \mathrm{Ci}$ ) of ${ }^{14} \mathrm{C}$-dasatinib (specific activity, $2.2 \mathrm{GBq} / \mathrm{mmol}$; Moravek Biochemicals) in $0.1 \mathrm{~mL}$ of vehicle (10\% Captisol [Cydex Pharmaceuticals, Inc.], $0.5 \% \mathrm{EtOH} /$ water) via the retroorbital venous plexus. The animals were sacrificed after $4 \mathrm{~h}$, and the glioblastomas were harvested, immediately fresh-frozen, and cut into $10-\mu \mathrm{m}$ sections. Autoradiography was performed by exposing slide-mounted sections on a BAS-MS 2325 phosphor imaging plate (FujiFilm Medical Systems USA, Inc.) for $12 \mathrm{~d}$; hematoxylin and eosin staining confirmed the presence of tumor. The imaging plate was scanned using a Typhoon FLA 7000 bioimaging analysis system (GE Healthcare Bio-Sciences) at a $25-\mu \mathrm{m}$-pixel size resolution with a pixel depth of 8 bit. Multi Gauge software (version 3.0; FujiFilm) analyzed the scanned images. Autoradiography was quantified with ImageJ by reporting the mean whole-tumor intensities of whole-tumor ROIs derived from corresponding hematoxylin- and eosinstained sections. Blood clearance of ${ }^{14} \mathrm{C}$-dasatinib was determined in naïve Ntva mice $(n=4)$. Blood was collected at $0.5,1,2,3$, and $4 \mathrm{~h}$ after injection, and all animals were sacrificed after $4 \mathrm{~h}$ and their brains harvested. Blood and brains were digested with Solvable solution (Perkin Elmer) and decolorized with hydrogen peroxide for scintillation counting. The percentage of injected dose per gram of tissue weight was calculated, and data were plotted as mean $\pm \mathrm{SE}$.

\section{Glioblastoma Neurosphere Dose Response to a- and y-Irradiation}

Primary culture neurospheres were obtained from PDGF-induced mouse glioblastomas. To determine the $\alpha$-irradiation geometry, confocal microscopy was performed on mechanically dissociated fixed and DAPI-stained neurosphere-derived cells plated at a concentration of 344 cells $/ \mathrm{mm}^{2}$ in the irradiator dish. The anodized aluminum irradiator dishes had a 1.5 - $\mu \mathrm{m}$-thick polyester thin-film exit window (Chemplex Industries). A TCS SP2 inverted confocal microscope (Leica) was used to obtain $\mathrm{z}$-stacked images at $\times 20$ magnification. The planar image was reconstructed using Imaris software. The average nuclear diameter was obtained from 10 nuclei. External $\alpha$-particle irradiation of dissociated neurosphere cells was performed as a function of dose. The irradiator (Biospherix) uses a ${ }^{241} \mathrm{Am}$ foil (NRD Inc.), which provided a source of collimated $\alpha$-particles at a flux of 792.8 particles $\mathrm{mm}^{-2} \mathrm{~s}^{-1}$. Neurospheres were mechanically dissociated into individual cells and diluted to a concentration of 100,000 cells $/ \mathrm{mL}$, and $2 \mathrm{~mL}$ of cells were added to each of 10 irradiator dishes. The settled cells were irradiated for $2.5,5,10,20,40,80,160$, or $320 \mathrm{~s}$. Two nonirradiated controls were included: the first was a nonirradiated growth control, and the second was $1 \mathrm{M} \mathrm{HCl}$ added. $\gamma$-irradiation was performed on similarly dissociated cells diluted to a concentration of 500 cells $/ \mathrm{mL}$, and $10 \mathrm{~mL}$ were placed in each of ten $15-\mathrm{mL}$ centrifuge tubes. The tubes were positioned in a fixed geometry and irradiated for $1.8,4.8,8.4,16.2,32.4,64.8,130.2,259.8$, or 324.6 s with a ${ }^{137} \mathrm{Cs}$ source (model 68; J.L. Shepherd and Associates) positioned to receive a dose rate of $1.85 \mathrm{~Gy} / \mathrm{min}$. After $\alpha$ - or $\gamma$-irradiation, the neurosphere assay was performed by adjusting the concentration to 500 cells $/ \mathrm{mL}$ per dose (clonal density) and plating $1 \mathrm{~mL}$ into each of 6 wells on a 12-well plate. An additional $0.5 \mathrm{~mL}$ of medium was added to each of the wells. Plated cells were placed in an incubator, and on the 6th day after irradiation the plates were removed and the number of neurospheres in each well was counted using a DM IRB microscope at $\times 5$ magnification (Leica). Dose-response curves were generated on the basis of the doses administered and the fraction of surviving cells.

\section{Statistical Analysis}

The Student $t$ test (unpaired, 2-tailed $t$ test) was used in Prism software for statistical analysis of data. Data are displayed as mean with SEM. $P$ values less than 0.05 were considered statistically significant.

\section{Study Approval}

All animal experiments were done in accordance with the guidelines of the National Institutes of Health on the care and use of laboratory animals and were approved by the Institutional Animal Care and Use Committee of Memorial Sloan Kettering Cancer Institute.

\section{RESULTS}

\section{Mechanism-of-Action Studies with ${ }^{225}$ Ac-E4G10 in Ntva Mouse Model}

The mechanism of ${ }^{225}$ Ac-E4G10 action was investigated in transgenic Ntva mice with glioblastoma. Ntva mice develop glioblastoma that models the high-grade proneural subtype in humans driven by overexpression of the oncogene PDGF (20). The animals were treated with one $7.4-\mathrm{kBq}$ dose of ${ }^{225} \mathrm{Ac}-\mathrm{E} 4 \mathrm{G} 104.5 \mathrm{wk}$ after glioblastoma induction, and therapeutic remodeling of the tumor microenvironment was analyzed $10 \mathrm{~d}$ later (Supplemental Fig. 1; supplemental materials are available at http://jnm.snmjournals. org). All mechanistic in vivo studies with ${ }^{225} \mathrm{Ac}-\mathrm{E} 4 \mathrm{G} 10$ were conducted using a ${ }^{225} \mathrm{Ac}$-isotype control antibody (rat $\mathrm{IgG} 2$ ) or vehicle control. Both antibodies were used at more than $97.5 \%$ radiochemical purity for the experiments (Supplemental Table 1).

\section{${ }^{225}$ AC-E4G10 Remodeling of the BBB Microenvironment in Glioblastoma Mice}

Glioblastoma sections of ${ }^{225}$ Ac-treated or control mice were imaged with immunofluorescence and immunohistochemistry markers to investigate morphologic changes in the tumor-BBB microenvironment. The normal BBB is a well-organized microenvironment that consists of tightly joined endothelial cells, pericytes, astrocytes, and basement membrane (23). We probed the aberrant glioblastoma-BBB architecture with immunofluorescence confocal microscopy. Tissue was costained with anti-CD31 antibodies (endothelial cells), anti-desmin antibodies (pericytes), anticollagen IV (basement membrane) antibodies, and DAPI (Fig. 1A and Supplemental Fig. 2). Vehicle-treated controls showed an extensive and irregular endothelial cell/pericyte margin with an unbalanced basement membrane structure. We found high pericyte coverage of blood vessels (desmin-to-CD31 signal ratio); the vascular endothelium was imbedded in a disorganized massive pericyte network (Figs. 1A-1C and Supplemental Fig. 2). ${ }^{225}$ Ac-E4G10 treatment resulted in a remodeled architecture. Whole-section quantification showed that overall cellularity was reduced and CD31 signal was significantly decreased $(P=0.036)$ versus vehicle control (Supplemental Figs. 3A and 3B). Collagen IV coverage of blood vessels was increased in ${ }^{225} \mathrm{Ac}$-treated animals (Supplemental Figs. 3C and 3D), yielding a more organized vasculature that was also less tortuous. In addition to depleting endothelial cells, ${ }^{225}$ Ac-E4G10 irradiation significantly reduced both pericyte density (Fig. 1B) and pericyte coverage (Fig. 1C) throughout the tumor, with $P$ equaling 0.048 and 0.049 , respectively. The number of pericytes associated with individual tumor vessels was less in ${ }^{225}$ Ac-E4G10-treated animals than in vehicle controls, as determined using reconstructed 3-dimensional images (Supplemental Figs. 4A and 4B).

The regulatory $\mathrm{T}$ cell population (FoxP3-positive) in the tumor microenvironment is reduced by ${ }^{225}$ Ac-E4G10 treatment (Figs. 1D and $1 \mathrm{E})$. Regulatory $\mathrm{T}$ cells are immunosuppressive and normally 


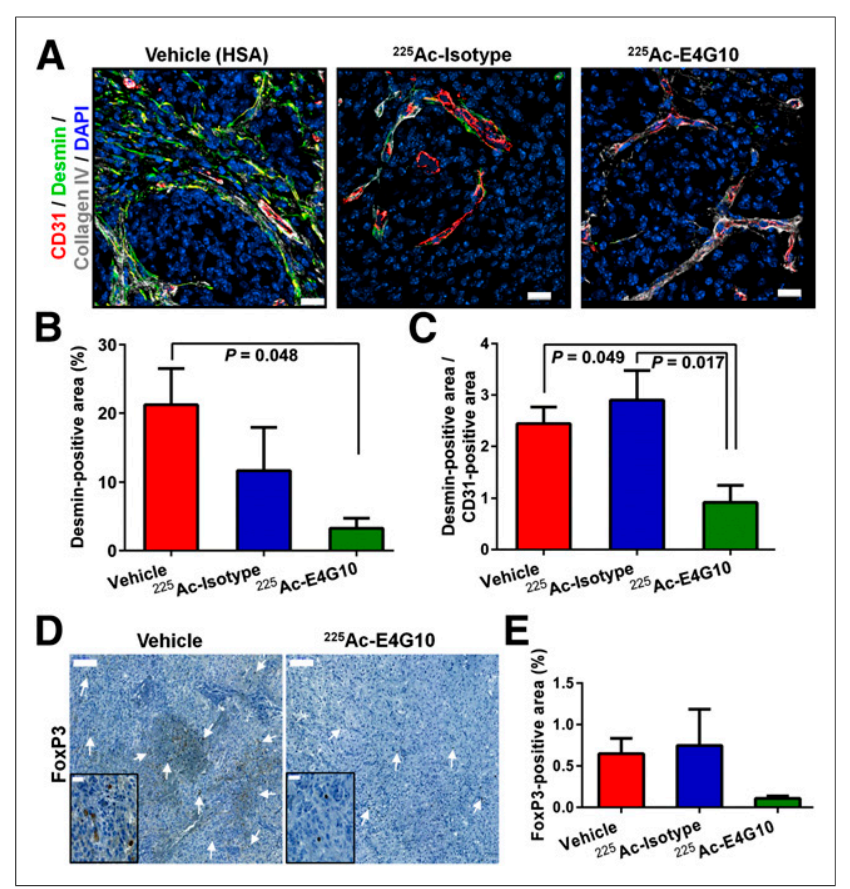

FIGURE 1. Histologic changes in glioblastoma microenvironment $10 \mathrm{~d}$ after ${ }^{225} \mathrm{Ac}-\mathrm{E} 4 \mathrm{G} 10$ treatment. (A) Confocal immunofluorescence imaging of tumor sections co-stained for anti-CD31, anti-desmin, and anti-collagen IV. Overlay of all stains is presented, with colocalization of endothelial cells (red) and pericytes (green) in yellow and counterstaining with DAPI (blue). Scale bars are $20 \mu \mathrm{m}$. (B and C) Quantification of pericyte density (desmin normalized by DAPI) (B) and pericyte coverage of blood vessels (desmin normalized by CD31) (C). (D) Immunohistochemical staining for regulatory T cells. Arrows indicate FoxP3-positive cells. (E) Quantification of FoxP3-positive regulatory T cells. Scale bars are $100 \mu \mathrm{m}$ (full panels) and $20 \mu \mathrm{m}$ (inset panels). Representative images of treatment and control groups are shown in A and D; positively stained area was counted and calculated as percentage of wholetumor section for quantifications shown in B, C, and E. Data are mean \pm SEM. HSA = human serum albumin.

prevent autoimmunity; however, they have been shown to suppress antitumor response and favor tumor progression in glioblastoma. We analyzed whole-tumor sections stained with an antibody specific for the transcription factor FoxP3, which is expressed by activated regulatory $\mathrm{T}$ cells. We found a greater than 6-fold decrease of infiltrating FoxP3-positive cells in the microenvironment of ${ }^{225}$ Ac-E4G10-treated versus vehicle-treated controls (Fig. 1E). We did not identify FoxP3-positive cells in healthy brain (Supplemental Fig. 5). Additionally, hypoxia and proliferation were analyzed in the bulk tumor. We found a trend toward reduced proliferation and hypoxia in ${ }^{225}$ Ac-E4G10-treated tumors compared with vehicletreated controls (Supplemental Figs. 6A and 6B).

\section{Increased Perfusion and Relief of Tumor-Associated Necrosis and Edema from ${ }^{225}$ Ac-E4G10 Treatment}

Diffusion-weighted MR imaging was performed to assess functional changes in transport phenomena due to $\alpha$-particle irradiation. We analyzed modifications in perfusion and diffusion parameters using diffusion-weighted MRI based on intravoxel incoherent motion (21). ROIs drawn over the entire tumor (Fig. 2A) and signal intensities were reported as a function of applied diffusion weightings (b-values). The signal intensity was plotted as a function of varying b-values (Fig. 2B). Diffusion-dependent ADC and $D$ (Figs. 2C and 2D) were lower in ${ }^{225} \mathrm{Ac}-\mathrm{E} 4 \mathrm{G} 10-$ treated mice than in vehicle-treated mice ( $P=0.089$ and $P=0.003$, respectively). Typically, increased tissue cellularity restricts diffusion and is associated with decreased $\mathrm{ADC}$ and $D$. However, glioblastoma cellularity after treatment was actually reduced compared with control (Supplemental Fig. 3A). In vehicle-treated glioblastoma mice, higher ADC and $D$ values were ascribed to increased necrosis and extracellular edema. ${ }^{225} \mathrm{Ac}-\mathrm{E} 4 \mathrm{G} 10$ resulted in an ADC decrease compared with vehicle controls $\left(1.65 \times 10^{-3} \mathrm{~mm}^{2} \mathrm{~s}^{-1}\right.$ vs. $\left.3.21 \times 10^{-3} \mathrm{~mm}^{2} \mathrm{~s}^{-1}\right) . D^{*}$ increased after ${ }^{225} \mathrm{Ac}-\mathrm{E} 4 \mathrm{G} 10$ compared with vehicle-treated controls (Fig. 2E); $D^{*}$ was 10 -fold greater in the specific treatment group than in the controls $\left(31.96 \times 10^{-3} \mathrm{~mm}^{2} \mathrm{~s}^{-1}\right.$ vs. $\left.3.89 \times 10^{-3} \mathrm{~mm}^{2} \mathrm{~s}^{-1}\right)$. The microenvironment remodeling that resulted from $\alpha$-particle irradiation improved functional perfusion.

\section{${ }^{225} \mathrm{AC}-\mathrm{E} 4 \mathrm{G} 10$ Enhancement of Penetration of ${ }^{14} \mathrm{C}$-Dasatinib into Glioblastoma}

The $\alpha$-particle-irradiated vascular microenvironment significantly increased uptake of a small-molecule drug into the tumor. Dasatinib is a multiple-tyrosine kinase inhibitor targeting the $\beta$-type PDGF receptor, SRC, c-kit, and several other tyrosine kinases frequently overexpressed in glioblastoma and has shown promising therapeutic results in preclinical models of glioblastoma (24). We investigated the glioblastoma penetration of ${ }^{14} \mathrm{C}$-labeled dasatinib using quantitative autoradiography. Activity-distribution histograms for whole-tumor sections displayed only minimal ${ }^{14} \mathrm{C}$ dasatinib signal in the vehicle-treated mice compared with the ${ }^{225}$ Ac-E4G10-treated mice (Fig. 3). The increase of dasatinib signal in the glioblastomas of mice treated with ${ }^{225} \mathrm{Ac}-\mathrm{E} 4 \mathrm{G} 10$ was

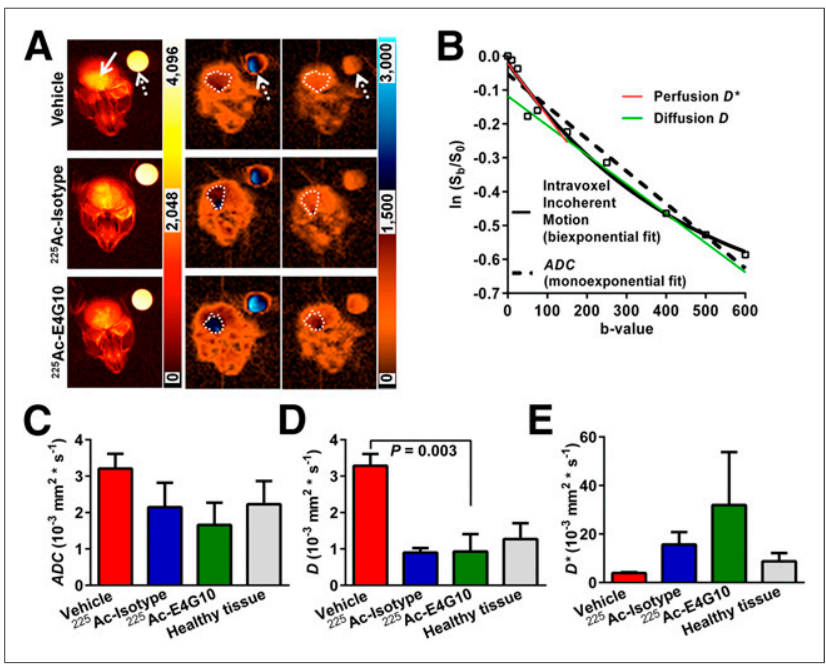

FIGURE 2. Diffusion-weighted MRI study of vessel functionality after ${ }^{225} \mathrm{Ac}$-E4G10 treatment. (A) Representative MR images on day 10 after treatment: axial T2-weighted fast spin echo (left); corresponding echoplanar at $b=50$ (predominantly perfusion) (middle); and echoplanar at $b=600$ (predominantly diffusion) (right). Scale bars indicate relative intensities; dotted outline, tumor margin; solid arrow, tumor; and dashed arrows, water-filled phantom (measurement control). (B) Representative logarithmic plot of signal intensities of whole-tumor ROls-plotted normalized to intensity at $b=0$ and as natural logarithm $\left(\ln \left(\mathrm{S}_{b} / S_{b=0}\right)\right)-v s$. measured b-values. Biexponential decay fitting of data (intravoxel incoherent motion, straight line) results in 2 slopes, $D^{*}$ (red line) and $D$ (green line). ADC is derived by monoexponential decay fitting of data (dashed line). (C-E) Quantification of coefficients: ADC (C), $D(\mathrm{D})$, and $D^{*}$ (E). Data are mean \pm SEM. 


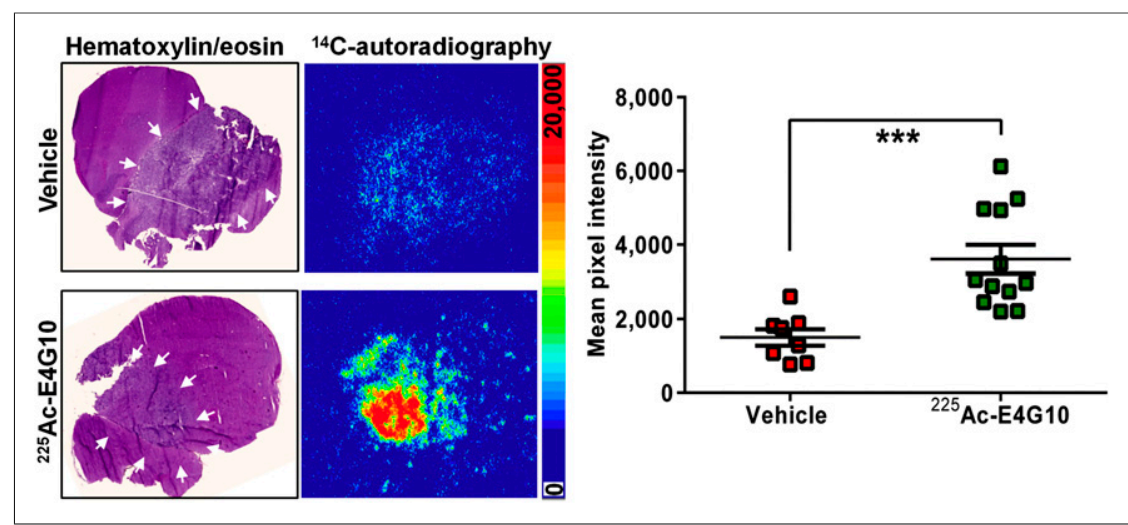

FIGURE 3. Dasatinib penetration of glioblastomas $10 \mathrm{~d}$ after ${ }^{225} \mathrm{Ac}-\mathrm{E} 4 \mathrm{G} 10$ treatment. Representative axial whole-brain sections stained with hematoxylin and eosin are shown, with matching autoradiography of ${ }^{14} \mathrm{C}$-labeled dasatinib from mice treated with vehicle $(n=5)$ or ${ }^{225} \mathrm{Ac}-\mathrm{E} 4 \mathrm{G} 10(n=6)$. Arrows indicate tumor margins. Scale bar indicates relative intensities. Autoradiography data are quantified on right. Data are mean \pm SEM of mean intensities of whole-tumor ROls. ${ }^{\star \star *} P<0.001$.

significantly increased, by $58 \%(P=0.0002)$. We attribute the increased uptake to improved drug delivery via the remodeled vascular network in conjunction with improved functionality. The blood clearance of ${ }^{14} \mathrm{C}$-dasatinib in Ntva mice was determined in order to confirm when radioactivity circulating in the blood pool was cleared sufficiently $(4 \mathrm{~h})$ for tissue harvesting (Supplemental Fig. 7).

\section{Depletion of EPCs in Bone Marrow of Ntva Mice}

The depletion of bone marrow EPCs by ${ }^{225}$ Ac-E4G10 also contributed to the mechanism of action by inhibiting vasculogenesis. Flow cytometry-based identification of EPC titer was used to evaluate population changes as a function of treatment (Fig. 4A and Supplemental Fig. 8). EPCs in the bone marrow are characterized by a VEGF receptor 2-positive/c-kit-positive/TER 119negative/CD11b-negative phenotype (22). These cells express

\section{DISCUSSION}

vascular endothelial cadherin (CD144) and therefore bind E4G10 (12). EPCs are produced in the bone marrow and released in response to injury, ischemia, and tumor growth (25). The bone marrow-EPC population increased from $0.16 \% \pm 0.02 \%$ in naïve mice to $0.26 \% \pm 0.03 \%$ in glioblastomabearing mice $(P=0.056$, Fig. 4B). A single dose of $7.4 \mathrm{kBq}$ of ${ }^{225} \mathrm{Ac}-\mathrm{E} 4 \mathrm{G} 10$ resulted in significant EPC depletion versus vehicle controls $(P=0.016$, Fig. $4 \mathrm{C})$.

\section{Sensitivity of Stemlike Glioblastoma Progenitor Cells to a-Particle Irradiation Ex Vivo}

Ex vivo experiments with tumor stemlike cells derived from glioblastoma neurospheres showed sensitivity to $\alpha$-irradiation $\left(D_{0}=0.25 \mathrm{~Gy}\right)$ versus $\gamma$-irradiation $\left(\mathrm{D}_{0}=\right.$ 1.65 Gy) as well as the absence of DNA repair (Supplemental Figs. 9 and 10). The potential for these cells to escape surgical resection, drug treatment, and external-beam irradiation may contribute to recurrent disease. Those stemlike progenitors that reside in the perivascular niche could be eradicated by collateral $\alpha$-particle irradiation.

One area of focus for improving the therapeutic options for glioblastoma is antivascular therapy, and one subject of our own investigations has been antivascular $\alpha$-particle therapy with ${ }^{225} \mathrm{Ac}$ E4G10. We recently reported increased survival and potent control of tumor growth from use of this drug as monotherapy in the clinically relevant Ntva model of high-grade glioblastoma (13). In the current study, we proposed a mechanism of action whereby the $\alpha$-particle irradiation of glioblastoma vascular endothelium would remodel the glioblastoma vascular microenvironment, and we found that $\alpha$-radioimmunotherapy produced changes in the morphology of the tumor-BBB microenvironment. We expected the reduction that we found in the endothelial cell population (48\% decrease); however, $\alpha$-particles also depleted the perivascular cell population. Pericyte density throughout the tumor and pericyte coverage were significantly reduced. Pericytes are located adjacent to endothelial cells and therefore within range of $\alpha$-particle emissions.

Dual targeting of vascular endothelium and pericytes has been shown to be highly effective for in vivo treatment of ovarian carcinoma, and several reports have demonstrated a beneficial antitumor effect in preclinical models if both the VEGF and the PDGF signaling axes are targeted, because signaling of the $\beta$-type PDGF receptor by pericytes is affected $(26,27)$. On the other hand, another outcome of tumor vessel normalization with antivascular treatment has been an increase in the perivascular coverage of vessels (28), as was
FIGURE 4. (A) Flow cytometry gating scheme to identify bone marrow EPCs in Ntva mice. After gating out debris (panel 1) and cell doublets based on forward scatter (panel 2), population of live (DAPI-negative) TER 119-negative and CD11b-negative cells is selected (third panel) and identified as VEGF receptor 2-positive, c-kit-positive EPCs (red square, panel 4). (B and C) Pretreatment (B) and posttreatment (C) comparisons of EPC population between Ntva mice and controls. Data are mean \pm SEM. FSC $=$ forward scatter; SSC $=$ side scatter. 
observed in a ${ }^{225} \mathrm{Ac}-\mathrm{E} 4 \mathrm{G} 10$-treated xenograft colon cancer model (10). However, pericyte coverage of tumor blood vessels varies in different tumor types and animal models (29). We observed an aberrantly high baseline pericyte coverage in untreated tumor vessels in the Ntva mouse model. Pericytes express PDGF receptors on their cell surface, and in Ntva mice, glioblastoma tumors are modeled under the background of PDGF overexpression, resulting in an aberrant pericyte network. $\alpha$-particle irradiation decreased this aberrant pericyte coverage in the remodeled microenvironment of Ntva mice. Pericytes play an important role in protecting endothelial cells, and it has been shown that one mechanism of resistance to therapy is promoted by increased pericyte coverage (30). ${ }^{225} \mathrm{Ac}-\mathrm{E} 4 \mathrm{G} 10$ could serve as a therapeutic option for resistance arising from conventional antivascular treatment options, especially in PDGF-overexpressing cancers.

Immunocytology showed that ${ }^{225} \mathrm{Ac}-\mathrm{E} 4 \mathrm{G} 10$ treatment depleted FoxP3-positive regulatory $\mathrm{T}$ cells. Regulatory $\mathrm{T}$ cells in the tumor microenvironment were decreased more than 6-fold compared with vehicle-treated controls. Glioblastoma is known to evade antitumor immune responses, with one of the escape mechanisms being infiltration of immunosuppressive regulatory $\mathrm{T}$ cells (24). Since recruitment and activation of regulatory $\mathrm{T}$ cells have a deleterious effect on antitumor immune response, the elimination of these cells is clinically highly desirable. It has been shown that intratumoral regulatory $\mathrm{T}$ cells correlate with tumor grade and that in vivo depletion of these cells significantly prolongs survival in mice with glioblastoma (31).

The observed depletion of pericytes and regulatory $\mathrm{T}$ cells in the tumor microenvironment suggests that $\alpha$-particles may also irradiate tumor stemlike cells residing in the perivascular niche. Ex vivo experiments with tumor stem cells derived from glioblastoma neurospheres showed these stemlike cells to have exquisite sensitivity to $\alpha$-irradiation $\left(\mathrm{D}_{0}=0.25 \mathrm{~Gy}\right)$ versus $\gamma$-irradiation $\left(\mathrm{D}_{0}=1.65 \mathrm{~Gy}\right)$, as well as the absence of DNA repair.

Hypoxia is a main driver of tumor progression, and some antiangiogenic therapies can increase hypoxia, leading to a more aggressive and infiltrative glioblastoma subtype after conventional antivascular treatments. Importantly, we did not observe any significant increase in either proliferation or hypoxia during the 10-d observation window after our application of antivascular ${ }^{225} \mathrm{Ac}-\mathrm{E} 4 \mathrm{G} 10$ treatment.

Improved vessel functionality after ${ }^{225} \mathrm{Ac}-\mathrm{E} 4 \mathrm{G} 10$ treatment was imaged and quantified using diffusion-weighted MRI. $D^{*}$ increased 10-fold after ${ }^{225}$ Ac-E4G10 treatment. An increase in the perfusion of treated vessels is a hallmark of antivascular treatments (32), especially for vessel-disrupting agents. We observed a significant reduction in the diffusion-dependent parameters $D$ and ADC. Restricted diffusion in glioblastoma is associated with increased cellularity and high-grade disease (33). However, our data showed decreased cellularity subsequent to ${ }^{225} \mathrm{Ac}$ treatment. We attribute the aberrantly high diffusion values for vehicle-treated animals to tumor-associated edema and necrotic areas. Therefore, the decreased diffusion in ${ }^{225}$ Ac-E4G10-treated samples is explained by reduced edema and necrosis. Our data from T2weighted fast spin echo imaging (Fig. 2A) support reduced edema, and the histology data show reduced necrosis. Vasogenic cerebral edema, and long-term use of corticosteroids to control edema, are a significant cause of morbidity and mortality in glioblastoma patients, with cerebral herniation seen in more than $60 \%(34,35)$. Treatment of glioblastoma-associated edema by the vessel-depleting agent cediranib was shown to be of major therapeutic benefit (35).
Any clinical advantage gained by reducing edema while avoiding corticosteroids would be desirable.

Significantly improved delivery of a small-molecule drug into the tumor results from enhanced vessel function. We measured significantly increased dasatinib uptake in ${ }^{225}$ Ac-E4G10-treated mice. Dasatinib has demonstrated promising preclinical results (24) but failed to show efficacy for recurrent glioblastoma in one phase II trial (36) or in combination with bevacizumab after bevacizumab failure in another phase II trial (37). The observed lack of response to dasatinib therapy was attributed to the inability of dasatinib to penetrate tumor in adequate concentrations. However, our quantitative autoradiographic data showed that ${ }^{225}$ Ac-E4G10 pretreatment improved uptake of dasatinib in glioblastoma.

We propose that the mechanism of ${ }^{225} \mathrm{Ac}-\mathrm{E} 4 \mathrm{G} 10$ action in the transgenic orthotopic glioblastoma model is a combination of vascular remodeling, edema relief, and regulatory $\mathrm{T}$ cell and EPC depletion. Tumor-BBB microenvironment remodeling was evidenced by the dual depletion of endothelial and perivascular cells. Tumor-associated edema and necrosis were relieved, as shown by increased perfusion and reduced overall diffusion parameters. Small-molecule drug uptake was also enhanced. We do not discount the possibility that irradiation of stemlike progenitor cells in the perivascular niche also has a role, but further investigation is necessary.

${ }^{225}$ Ac-E4G10 offers a unique antivascular approach for glioblastoma treatment. First, the targeted endothelial cells are genetically stable but vulnerable to $\alpha$-particle irradiation (38). Second, ${ }^{225}$ Ac-E4G10 does not work by blocking of proangiogenic factors but by cytotoxic depletion of the specific cell populations that signal using these proangiogenic factors in the tumor microenvironment; hence, the tumor cannot evade therapy by activation or upregulation of alternative signaling pathways. Third, ${ }^{225} \mathrm{Ac}-$ E4G10 treatment also inhibits vasculogenesis, limiting a process shown to obviate VEGF signaling (39). Fourth, ${ }^{225}$ Ac-E4G10 treatment co-depletes pericytes, recognized promoters of resistance to antiangiogenic treatments. ${ }^{225}$ Ac-lintuzumab has shown clinical promise to treat leukemia and has been safely used with no significant toxicities (40).

\section{CONCLUSION}

We have shown previously that ${ }^{225}$ Ac-E4G10 potently controls tumor growth and extends survival in mice with glioblastoma. The current study showed that targeted antivascular $\alpha$-particle radiation remodels the glioblastoma vascular microenvironment via a multimodal mechanism of action and provides insight into the vascular architecture of platelet-derived growth factor-driven glioblastoma. Translation into the clinic to treat recurrent glioblastoma should be considered.

\section{DISCLOSURE}

The costs of publication of this article were defrayed in part by the payment of page charges. Therefore, and solely to indicate this fact, this article is hereby marked "advertisement" in accordance with 18 USC section 1734 . This work was supported by grants R01 CA166078, R01 CA55349, R25T CA046945, R24 CA83084, P30 CA08748, P01 CA33049, F31 CA167863, and R00 EB014328 from the NIH; the MSK Experimental Brain Tumor Center; Mr. William H. and Mrs. Alice Goodwin and the Commonwealth Foundation for Cancer Research; the Center for Experimental 
Therapeutics of MSKCC; and the MSK Center for Molecular Imaging and Nanotechnology. Eli Lilly and Company provided the E4G10 antibody, and Actinium Pharmaceuticals, Inc., the ${ }^{225}$ Ac. Drs. McDevitt and Scheinberg declare associations with Actinium Pharmaceuticals, Inc. No other potential conflict of interest relevant to this article was reported.

\section{ACKNOWLEDGMENTS}

We thank Drs. Eric Holland and Johanna Joyce for the Ntva breeding pairs, Dr. Aneesh Sheth for technical support, and the MSKCC Molecular Cytology Core Facility.

\section{REFERENCES}

1. Shih T, Lindley C. Bevacizumab: an angiogenesis inhibitor for the treatment of solid malignancies. Clin Ther. 2006;28:1779-1802.

2. Folkman J. What is the evidence that tumors are angiogenesis dependent? J Natl Cancer Inst. 1990;82:4-6.

3. Siemann DW. The unique characteristics of tumor vasculature and preclinical evidence for its selective disruption by tumor-vascular disrupting agents. Cancer Treat Rev. 2011;37:63-74.

4. Gotink KJ, Verheul HM. Anti-angiogenic tyrosine kinase inhibitors: what is their mechanism of action? Angiogenesis. 2010;13:1-14.

5. Lu KV, Bergers G. Mechanisms of evasive resistance to anti-VEGF therapy in glioblastoma. CNS Oncol. 2013;2:49-65.

6. Siemann DW. Therapeutic strategies that selectively target and disrupt established tumor vasculature. Hematol Oncol Clin North Am. 2004;18:1023-1037.

7. McDevitt MR, Sgouros G, Finn RD, et al. Radioimmunotherapy with alphaemitting nuclides. Eur J Nucl Med. 1998;25:1341-1351.

8. McDevitt MR, Ma D, Lai LT, et al. Tumor therapy with targeted atomic nanogenerators. Science. 2001;294:1537-1540.

9. Singh Jaggi J, Henke E, Seshan SV, et al. Selective alpha-particle mediated depletion of tumor vasculature with vascular normalization. PLoS One. 2007; 2:e267.

10. Escorcia FE, Henke E, McDevitt MR, et al. Selective killing of tumor neovasculature paradoxically improves chemotherapy delivery to tumors. Cancer Res. 2010;70:9277-9286.

11. Corada M, Zanetta L, Orsenigo F, et al. A monoclonal antibody to vascular endothelial-cadherin inhibits tumor angiogenesis without side effects on endothelial permeability. Blood. 2002;100:905-911.

12. Nolan DJ, Ciarrocchi A, Mellick AS, et al. Bone marrow-derived endothelial progenitor cells are a major determinant of nascent tumor neovascularization. Genes Dev. 2007;21:1546-1558.

13. Behling K, Maguire WF, López Puebla JC, et al. Vascular targeted radioimmunotherapy for the treatment of glioblastoma. J Nucl Med. 2016

14. Louis DN, Ohgaki H, Wiestler OD, et al. The 2007 WHO classification of tumours of the central nervous system. Acta Neuropathol (Berl). 2007;114: 97-109.

15. Jain RK, di Tomaso E, Duda DG, Loeffler JS, Sorensen AG, Batchelor TT. Angiogenesis in brain tumours. Nat Rev Neurosci. 2007;8:610-622.

16. Wolburg H, Noell S, Fallier-Becker P, Mack AF, Wolburg-Buchholz K. The disturbed blood-brain barrier in human glioblastoma. Mol Aspects Med. 2012; 33:579-589.

17. Pham K, Luo D, Siemann DW, et al. VEGFR inhibitors upregulate CXCR4 in VEGF receptor-expressing glioblastoma in a TGFbetaR signaling-dependent manner. Cancer Lett. 2015;360:60-67.
18. Maguire WF, McDevitt MR, Smith-Jones PM, Scheinberg DA. Efficient 1-step radiolabeling of monoclonal antibodies to high specific activity with ${ }^{225} \mathrm{Ac}$ for alpha-particle radioimmunotherapy of cancer. J Nucl Med. 2014;55:1492-1498.

19. McDevitt MR, Ma D, Simon J, Frank RK, Scheinberg DA. Design and synthesis of ${ }^{225}$ Ac radioimmunopharmaceuticals. Appl Radiat Isot. 2002;57:841-847.

20. Hambardzumyan D, Amankulor NM, Helmy KY, Becher OJ, Holland EC. Modeling adult gliomas using RCAS/t-va technology. Transl Oncol. 2009;2:89-95.

21. Le Bihan D, Breton E, Lallemand D, Aubin ML, Vignaud J, Laval-Jeantet M. Separation of diffusion and perfusion in intravoxel incoherent motion MR imaging. Radiology. 1988;168:497-505.

22. Penack O, Henke E, Suh D, et al. Inhibition of neovascularization to simultaneously ameliorate graft-vs-host disease and decrease tumor growth. $J$ Natl Cancer Inst. 2010;102:894-908.

23. Abbott NJ, Patabendige AA, Dolman DE, Yusof SR, Begley DJ. Structure and function of the blood-brain barrier. Neurobiol Dis. 2010;37:13-25.

24. Ahluwalia MS, de GJ, Liu WM, Gladson CL. Targeting SRC in glioblastoma tumors and brain metastases: rationale and preclinical studies. Cancer Lett. 2010;298:139-149.

25. Yoder MC. Human endothelial progenitor cells. Cold Spring Harb Perspect Med. 2012;2:a006692

26. Lu C, Kamat AA, Lin YG, et al. Dual targeting of endothelial cells and pericytes in antivascular therapy for ovarian carcinoma. Clin Cancer Res. 2007;13:42094217.

27. Shen J, Vil MD, Zhang H, et al. An antibody directed against PDGF receptor beta enhances the antitumor and the anti-angiogenic activities of an anti-VEGF receptor 2 antibody. Biochem Biophys Res Commun. 2007;357:1142-1147.

28. Goel S, Duda DG, Xu L, et al. Normalization of the vasculature for treatment of cancer and other diseases. Physiol Rev. 2011;91:1071-1121.

29. Morikawa S, Baluk P, Kaidoh T, Haskell A, Jain RK, McDonald DM. Abnormalities in pericytes on blood vessels and endothelial sprouts in tumors. Am J Pathol. 2002;160:985-1000.

30. Bergers G, Song S, Meyer-Morse N, Bergsland E, Hanahan D. Benefits of targeting both pericytes and endothelial cells in the tumor vasculature with kinase inhibitors. J Clin Invest. 2003;111:1287-1295.

31. El Andaloussi A, Han Y, Lesniak MS. Prolongation of survival following depletion of $\mathrm{CD} 4+\mathrm{CD} 25+$ regulatory $\mathrm{T}$ cells in mice with experimental brain tumors. J Neurosurg. 2006;105:430-437.

32. Jain RK. Normalizing tumor microenvironment to treat cancer: bench to bedside to biomarkers. J Clin Oncol. 2013;31:2205-2218.

33. Zulfiqar M, Yousem DM, Lai H. ADC values and prognosis of malignant astrocytomas: does lower ADC predict a worse prognosis independent of grade of tumor? A meta-analysis. AJR. 2013;200:624-629.

34. Gerstner ER, Duda DG, di Tomaso E, et al. VEGF inhibitors in the treatment of cerebral edema in patients with brain cancer. Nat Rev Clin Oncol. 2009;6:229236 .

35. Kamoun WS, Ley CD, Farrar CT, et al. Edema control by cediranib, a vascular endothelial growth factor receptor-targeted kinase inhibitor, prolongs survival despite persistent brain tumor growth in mice. J Clin Oncol. 2009;27:2542-2552.

36. Lassman AB, Pugh SL, Gilbert MR, et al. Phase 2 trial of dasatinib in targetselected patients with recurrent glioblastoma (RTOG 0627). Neuro-Oncol. 2015;17:992-998.

37. Lu-Emerson C, Norden AD, Drappatz J, et al. Retrospective study of dasatinib for recurrent glioblastoma after bevacizumab failure. J Neurooncol. 2011; 104:287-291.

38. Kerbel R, Folkman J. Clinical translation of angiogenesis inhibitors. Nat Rev Cancer. 2002;2:727-739.

39. Bergers G, Hanahan D. Modes of resistance to anti-angiogenic therapy. Nat Rev Cancer. 2008;8:592-603.

40. Sgouros G, ed. MIRD Radiobiology and Dosimetry for Radiopharmaceutical Therapy with Alpha-Particle Emitters. Reston, VA: Society of Nuclear Medicine and Molecular Imaging; 2015. 